\title{
The covariant description of electric and magnetic field lines of null fields: application to Hopf-Rañada solutions
}

\author{
S.J. van $\mathrm{Enk}^{1,2}$ \\ ${ }^{1}$ Physics Department and Oregon Center for Optics, \\ University of Oregon, Eugene, OR 97403, USA and \\ ${ }^{2}$ Huygens Laboratory of Physics, University of Leiden, 2333 CA Leiden, The Netherlands
}

\begin{abstract}
The concept of electric and magnetic field lines is intrinsically non-relativistic. Nonetheless, for certain types of fields satisfying certain geometric properties, field lines can be defined covariantly. More precisely, two Lorentz-invariant 2D surfaces in spacetime can be defined such that magnetic and electric field lines are determined, for any observer, by the intersection of those surfaces with spacelike hyperplanes. An instance of this type of field is constituted by the so-called Hopf-Rañada solutions of the source-free Maxwell equations, which have been studied because of their interesting topological properties, namely, linkage of their field lines. In order to describe both geometric and topological properties in a succinct manner, we employ the tools of Geometric Algebra (aka Clifford Algebra) and use the Clebsch representation for the vector potential as well as the Euler representation for both magnetic and electric fields. This description is easily made covariant, thus allowing us to define electric and magnetic field lines covariantly in a compact geometric language. The definitions of field lines can be phrased in terms of 2D surfaces in space. We display those surfaces in different reference frames, showing how those surfaces change under Lorentz transformations while keeping their topological properties. As a byproduct we also obtain relations between optical helicity, optical chirality and generalizations thereof, and their conservation laws.
\end{abstract}

\section{INTRODUCTION}

The concept of field lines in electromagnetics (EM) is interesting for various reasons. Historically, Faraday considered field lines as the fundamental entities of EM (see, e.g., Chapter 3 of Ref. [1). Moreover, everyone's first acquaintance with the concept of magnetic field is, probably, through the simple experiment with iron filings that makes magnetic field lines around a bar magnet visible. On the other hand, by the time one is an advanced student learning about Special Relativity (SR) and the role Maxwell's equations played in its development, one typically does not hear much anymore about field lines. And indeed, the concept of field lines is highly non-relativistic for two reasons. First, electric and magnetic fields transform into each other under Lorentz transformations, and second, the points on a field line all have the same time coordinate.

As it turns out, one can define electric and magnetic field lines in a relativistically covariant manner only for certain types of solutions of Maxwell's equations. This has been known for magnetic field lines since a seminal paper by Newcomb 2] (and as a demonstration of current interest in the covariant description of magnetic field lines, see [? ]). There he also discusses two related concepts that are logically prior to covariance of field lines, namely, identity of field lines and motion of field lines. For, in order to discuss what a given field line for one observer looks like to another observer, one needs to be able to identify the field line in the first place. Similarly, even for one fixed observer, in order to be able to talk about the motion of a given field line one needs to identify which field lines at two different times are deemed to be the same. As Newcomb derived in Ref. 2], magnetic field lines can be said to move with a (position and time-dependent) velocity $\vec{v}$ if and only if (employing units where $c=1$ )

$$
\vec{\nabla} \times(\vec{E}+\vec{v} \times \vec{B})=0 .
$$

He further derived that a covariant description of magnetic field lines exists if and only if

$$
\vec{E} \cdot \vec{B}=0
$$

We will indicate how these conditions arise in Sections $\Pi$ and $\mathrm{V}$, respectively. In the special case that (2) is satisfied, we can set $\vec{v}=\vec{E} \times \vec{B} / \vec{B}^{2}$ to satisfy (1) identically. In the even more special case where $\vec{E} \cdot \vec{B}=0$ and $\vec{E}^{2}=\vec{B}^{2}$, we have $|\vec{v}|=1$, i.e., the magnetic field lines move with the speed of light. Such fields are called "null fields' and they are the subject of study in this paper.

Not all representations of the EM field are equally well suited for discussing and making covariant the concept of field lines. For example, the standard representation of the magnetic field consists of specifying $\vec{B}(\vec{r}, t)$ as a function of position $\vec{r}$ and time $t$. The magnetic field line at time $t_{0}$ passing through a given point $\vec{r}_{0}$ is then a parametrized curve $\vec{f}(\lambda)$ found, in principle, by solving the differential equation

$$
\frac{\mathrm{d} \vec{f}(\lambda)}{\mathrm{d} \lambda}=\frac{\vec{B}\left(\vec{f}(\lambda), t_{0}\right)}{\left|\vec{B}\left(\vec{f}(\lambda), t_{0}\right)\right|}
$$

with "initial" condition $\vec{f}(0)=\vec{r}_{0}$. Instead, we will make use of a more convenient representation, which does not require solving differential equations. When applied to the magnetic field, the representation goes under the name of Euler potentials 3. It leads naturally to a particular representation of the vector potential developed by Clebsch. This representation has several advantages in the present context: (i) it allows one to directly define magnetic field 
lines, (ii) it is easily made covariant for certain types of fields, (iii) it shows that field lines can be linked only if the gradient of one of the potentials is singular, and (iv) in it, the magnetic helicity, a quantity known to contain topological information about the linkage of magnetic field lines [4] [6], is compactly expressed. These advantages will be exploited here as we are interested in displaying topological properties possessed by the (magnetic and electric) field lines of so-called Hopf-Rañada solutions 7-11. These are solutions to the free Maxwell equations (without charges and currents). In order to achieve the same compactness and simplicity in describing their electric field lines, we make use of the dual symmetry of the free Maxwell equations. In standard notation this symmetry transformation is

$$
\begin{aligned}
& \vec{E} \mapsto \cos \theta \vec{E}+\sin \theta \vec{B}, \\
& \vec{B} \mapsto \cos \theta \vec{B}-\sin \theta \vec{E} .
\end{aligned}
$$

This symmetry, and the geometric conditions a field has to satisfy in order to be able to define both electric and magnetic field lines, as well as covariance [not to mention Maxwell's equations], all turn out to be easily and much more compactly expressed within the formalism of Geometric Algebra (GA) [12 16. Because this formalism is not widely known (yet), we will start with standard noncovariant vector calculus and use it to define Euler potentials and field lines in Section III, and only then will we introduce and summarize the most relevant aspects of GA in Section 【II. That formalism is used to describe EM noncovariantly in Section IV] (with conservation laws of helicity and like quantities easily obtained), followed by the covariant description in Section $\mathrm{V}$. All that theory is applied to Hopf-Rañada solutions and a description of their field lines in Section VI. In general, it is not easy to obtain Euler potentials for a given solution, but Rañada has provided explicit expressions for Euler potentials in his articles [7, 8, about the Hopf-Rañada solutions. In addition, those articles contain many deep insights, only some of which are exploited here. In Section VII we consider a much broader class of solutions to the free Maxwell equations, obtained long ago by Bateman [17, for which a covariant description of field lines is in principle possible. We give these solutions in an elegant covariant form, without, however, being able to find the covariant Euler potentials.

\section{EULER POTENTIALS AND THE CLEBSCH REPRESENTATION}

\section{A. Euler potentials}

The Euler representation of the magnetic field amounts to writing (for historical and mathematical background, see [3])

$$
\vec{B}=\vec{\nabla} \alpha \times \vec{\nabla} \beta,
$$

where $\alpha$ and $\beta$ are two scalar functions of position $\vec{r}$ and time $t$. (Euler used this representation not for magnetic fields, of course, but for incompressible fluid flows, described by a flow velocity $\vec{v}_{f}$ with $\vec{\nabla} \cdot \vec{v}_{f}=0$.) The advantages of representing $\vec{B}$ by Eq. (5) are that (i) the constraint

$$
\vec{\nabla} \cdot \vec{B}=0
$$

holds automatically if $\alpha$ and $\beta$ are sufficiently smooth functions of position, (ii) it conforms to the fact that in principle two scalar functions should suffice to express the three components of $\vec{B}$ subject to the single scalar constraint (6), and (iii) magnetic field lines at a given time $t$ are tangential to surfaces of constant $\alpha$ and to surfaces of constant $\beta$, and are thus determined by setting $\alpha$ and $\beta$ at time $t$ equal to constants, say,

$$
\begin{aligned}
\alpha(\vec{r}, t) & =\alpha_{0}, \\
\beta(\vec{r}, t) & =\beta_{0} .
\end{aligned}
$$

Generically, the locus of points $\vec{r}$ satisfying these two constraints defines a $1 \mathrm{D}$ curve in $3 \mathrm{D}$ space, and that curve is a field line of $\vec{B}$.

A disadvantage is that the Euler potentials are far from unique. In fact, it is clear that any two functions $f(\alpha, \beta)$ and $g(\alpha, \beta)$ determine the same magnetic field lines (and thereby the same $\vec{B}$ field) as long as the equations

$$
\begin{aligned}
& f(\alpha, \beta)=f\left(\alpha_{0}, \beta_{0}\right) \\
& g(\alpha, \beta)=g\left(\alpha_{0}, \beta_{0}\right),
\end{aligned}
$$

have unique solutions for $\alpha$ and $\beta$. This will be the case whenever the Jacobian for the transformation $(\alpha, \beta) \mapsto$ $(f, g)$

$$
J=\left|\begin{array}{ll}
\partial f / \partial \alpha & \partial g / \partial \alpha \\
\partial f / \partial \beta & \partial g / \partial \beta
\end{array}\right|
$$

is never zero anywhere. More precisely, in that case we have

$$
\vec{B}=J^{-1} \vec{\nabla} f \times \vec{\nabla} g,
$$

so that new Euler potentials $(f, g)$ are produced by any transformation $(\alpha, \beta) \mapsto(f, g)$ with $J \equiv 1$. To demonstrate the disadvantage of the non-uniqueness, it suffices to note that, if only the Euler potentials were unique, then field lines could be given an identity straightforwardly through the use of (7), by identifying a field line at all times by the pair of constants $\left(\alpha_{0}, \beta_{0}\right)$. In turn this would immediately yield a (position- and time-dependent) velocity $\vec{v}$ of the magnetic field lines. But as it is, one has to do a little bit more work to obtain a consistent definition of a field line velocity $\vec{v}$. Let us assume that field lines can be assigned a field line velocity. In particular, assume there is a vector field $\vec{v}$ such that the time evolution of a pair of Euler potentials $(\alpha, \beta)$ satisfies

$$
\begin{aligned}
& \partial_{t} \alpha+\vec{v} \cdot \vec{\nabla} \alpha=0, \\
& \partial_{t} \beta+\vec{v} \cdot \vec{\nabla} \beta=0 .
\end{aligned}
$$


Then, in order to obtain equations for the physical field $\vec{B}$ we multiply these two equations by the gradients of $\beta$ and $\alpha$, respectively, and then subtract the two. This yields [3]

$$
\vec{v} \times \vec{B}=\partial_{t} \alpha \vec{\nabla} \beta-\partial_{t} \beta \vec{\nabla} \alpha .
$$

We have not encountered the quantity appearing on the right-hand side yet, but the well-trained eye will recognize that it, together with $\vec{\nabla} \alpha \times \vec{\nabla} \beta$, will form a Lorentz covariant object (see Eq. (78) below). Taking the curl of (12) gives us an equation independent of the Euler representation:

$$
\vec{\nabla} \times(\vec{v} \times \vec{B})=\partial_{t} \vec{B} .
$$

Since we are interested in solutions to the source-free Maxwell equations, we can replace the term on the righthand side by $-\vec{\nabla} \times \vec{E}$, thus arriving at

$$
\vec{\nabla} \times(\vec{E}+\vec{v} \times \vec{B})=0,
$$

which is the (necessary and sufficient) condition Newcomb [2] found for a velocity $\vec{v}$ to be interpretable as a magnetic field line velocity.

\section{B. Clebsch representation and helicity}

Given Euler potentials $\alpha$ and $\beta$ it may seem straightforward now to define a vector potential $\vec{A}$ from which $\vec{B}$ can be derived as $\vec{B}=\vec{\nabla} \times \vec{A}$, namely by

$$
\vec{A}=-\beta \vec{\nabla} \alpha .
$$

There is, however, a subtlety associated with the magnetic helicity $H$ of the field, as has been discussed recently in, e.g., Ref. [18, as well as long ago in [19, and by Rañada in the context of the Hopf-Rañadasolutions in [6]. The magnetic helicity density $h_{m}$ is defined as

$$
h_{m}=\vec{A} \cdot \vec{B},
$$

in terms of which the magnetic helicity is given as an integral over all space

$$
H_{m}=\iiint h_{m}(\vec{r}) \mathrm{d} \vec{r} .
$$

The form 15) implies that $h_{m}=0$, and hence $H_{m}=0$. The next question is whether $H_{m}$ is gauge-invariant $\left(h_{m}\right.$ is certainly not!). Under a gauge transformation $\delta \vec{A}=\vec{\nabla} \psi$, we get, assuming we can apply Gauss's theorem,

$$
\delta H_{m}=\iiint(\vec{\nabla} \psi \cdot \vec{B}) \mathrm{d} \vec{r}=\iint\left(\psi \vec{B} \cdot \vec{e}_{n}\right) \mathrm{d} S
$$

where the latter integral is a surface integral, with $\vec{e}_{n}$ denoting the unit vector normal to the surface. For fields such that $|\vec{B}| \rightarrow 0$ sufficiently fast for $r \rightarrow \infty$, with $r=|\vec{r}|$, we have that $\delta H_{m}=0$. For the solutions we will consider the magnetic field does decay sufficiently fast, and so $H_{m}$ is gauge-invariant. In particular, it may now seem that, in fact, $H_{m}=0$ for all such fields. One interesting aspect of the Hopf-Rañada solutions, however, is that we cannot so simply apply Gauss's theorem. In particular, one of the Euler potentials - and we pick $\alpha$ here - must be multivalued, and its gradient singular. More precisely, $\alpha$ will be given in terms of a particular complex function $\eta$ (to be defined below) as

$$
\alpha=\frac{1}{2 \pi} \arctan \left(\frac{\operatorname{Re}(\eta)}{\operatorname{Im}(\eta)}\right) .
$$

This defines a multi-valued function, whose gradient is singular in the locus of points $L$ where $\eta=0$, since

$$
\vec{\nabla} \alpha=\frac{\operatorname{Im}(\eta) \vec{\nabla} \operatorname{Re}(\eta)-\operatorname{Re}(\eta) \vec{\nabla} \operatorname{Im}(\eta)}{2 \pi|\eta|^{2}} .
$$

Eq. 15 implies that $\vec{A}$ would be singular, too, at the locus $L$. However, we can choose a non-singular vector potential by adding a gauge-like term that cancels the singularity of the first term, viz.

$$
\vec{A}=-\beta \vec{\nabla} \alpha+\vec{\nabla} \Psi
$$

provided we pick $\Psi$ multivalued as well, according to

$$
\Psi=\beta(L) \alpha,
$$

where $\beta(L)$ is the value (presumed unique) attained by $\beta$ in the locus of points $L$. If $\beta$ is determined by $|\eta|^{2}$ alone (which is true for the Hopf-Rañada solutions to be discussed below) $\beta$ takes on a unique value $\beta(L)$ in all points of the locus $L$. The helicity $H_{m}$ can now be nonzero by virtue of the multi-valued character of both $\Psi$ and $\alpha$ (and this singularity is, therefore, necessary in order to describe fields with linked field lines [4, 5]). In fact, we have

$$
\begin{aligned}
H_{m} & =\iiint(\vec{\nabla} \alpha \times \vec{\nabla} \beta) \cdot \vec{\nabla} \Psi \mathrm{d} \vec{r} \\
& =\beta(L)\left(\beta_{\max }-\beta_{\min }\right) n^{2}\left(\alpha_{\max }-\alpha_{\min }\right)^{2},
\end{aligned}
$$

where $n$ is an integer quantifying the type of multivaluedness of $\alpha$ and $\Psi$ : it counts the number of branches of the function $\alpha$. For the standard Hopf-Rañada solution we have $n=1$. This expression makes manifest the precise relation between magnetic helicity and the Euler potentials.

\section{GEOMETRIC ALGEBRA}

Here we will briefly review the subject of Geometric Algebra (GA), also known as Clifford Algebra. Extensive introductions can be found in articles by Hestenes [12, 13, who has been advocating its use in physics over many years, as well as in the two textbooks Refs. [14, 15, and in a book of lecture notes [16].

One could say that the idea of GA is to subsume both the dot product of (3D) vectors and their cross product under a single vector product that avoids certain shortcomings 
of the cross product, but it comes with a multitude of additional benefits, some of which will be made use of here. The shortcomings of the cross product are that (i) it is not associative and (ii) the geometric notion that $\vec{a} \times \vec{b}$ is a vector pointing in the unique (up to a sign) direction perpendicular to the plane spanned by $\vec{a}$ and $\vec{b}$ does not generalize to higher dimensions. The vector product of GA is associative and its geometric meaning does generalize to any number of dimensions, for example, to the 4 dimensions of spacetime. Now it turns out that an elegant covariant description is possible using the GA of $3 \mathrm{D} \mathrm{Eu-}$ clidean space $\mathbb{E}^{3}$. That is, we do not need the GA of $4 \mathrm{D}$ Minkowski space, for reasons discussed in great detail in the book [14] and articles by Baylis 20 22] (see also below). We will, therefore, focus here on the GA of $\mathbb{E}^{3}$. We denote that algebra by $\mathcal{C}_{3}$ ( $\mathrm{C}$ for Clifford).

The vector product of two vectors $\vec{a}$ and $\vec{b}$ is constructed to be associative and to obey distributive laws for addition and multiplication. An additional axiom is that the vector product of any vector with itself equal the length squared of the vector

$$
\vec{a} \vec{a}=|\vec{a}|^{2} .
$$

The algebra is then built up from the set of all vectors by repeatedly taking sums and products of vectors. We can in the end distinguish four (always one more than the dimension of the underlying vector space) different geometric types of basis elements. First, we have the standard vectors of $\mathbb{E}^{3}$. Second, we encounter scalars, because of (24). The third type arises when we consider the product of two linearly independent vectors, say, $\vec{a}$ and $\vec{b}$. Their product splits into a commuting symmetric scalar term $\vec{a} \cdot \vec{b}$ and an anti-commuting anti-symmetric "bivector" term (our third type of term) denoted by $\vec{a} \wedge \vec{b}$, the wedge product:

$$
\vec{a} \vec{b}=\frac{1}{2}(\vec{a} \vec{b}+\vec{b} \vec{a})+\frac{1}{2}(\vec{a} \vec{b}-\vec{b} \vec{a})=\vec{a} \cdot \vec{b}+\vec{a} \wedge \vec{b} .
$$

The wedge product represents an oriented area for the plane spanned by the two vectors. Moreover, it generates rotations in that plane (both these meanings easily generalize to more dimensions). More precisely, if we use an orthonormal basis $\left\{\vec{e}_{1}, \vec{e}_{2}\right\}$ for a given plane, then any vector $\vec{c} \in \mathbb{E}^{3}$ is rotated in the plane by an angle $\theta$ by the transformation

$$
\vec{c} \mapsto \vec{c}^{\prime}=\mathrm{R} \vec{c} \mathrm{R}^{\dagger}
$$

with

$$
\mathrm{R}=\exp \left(\frac{1}{2} \theta \vec{e}_{2} \vec{e}_{1}\right)
$$

and where the $\dagger$ operation reverses the order of vectors in any product. One should note here that $\left(\vec{e}_{2} \vec{e}_{1}\right)^{2}=-1$, so that we can also write

$$
\mathrm{R}=\cos \left(\frac{1}{2} \theta\right)+\sin \left(\frac{1}{2} \theta\right) \vec{e}_{2} \vec{e}_{1}
$$

This shows explicitly that $\mathrm{R}$ is a sum of two different types of elements, a scalar and a bivector, and such elements of mixed type will always be denoted by sans serif symbols.
A product of three vectors can be expanded in vector terms and a "trivector" term, consisting of a product of three orthogonal vectors. (This is the fourth and last type of term we encounter.) When we take those vectors to be unit vectors forming a right-handed frame, then we denote the resulting trivector by

$$
I=\vec{e}_{1} \vec{e}_{2} \vec{e}_{3} .
$$

One can easily verify that this entity is the same for any right-handed set of orthogonal vectors. Moreover, it satisfies

$$
I^{2}=-1,
$$

and it commutes with all bivectors and all vectors. It thus plays a very similar role as the standard imaginary unit $i$ (and it is denoted as such, by $i$, in many papers and books on GA; here we keep a different notation, to make sure we remember $I$ is, in fact, a trivector). The geometric meaning of $I$ is that it represents an oriented volume, spanned by the three basis vectors (this geometric meaning makes it obvious there is only one such element in $3 \mathrm{D}$, whereas in higher dimensions there exist multiple linearly independent trivectors). $I$ is a pseudoscalar as it changes sign under parity reversal. Importantly, in $3 \mathrm{D}$, the standard cross product is related to the wedge product by

$$
\vec{a} \wedge \vec{b}=I \vec{a} \times \vec{b}
$$

In words, the bivector $\vec{a} \wedge \vec{b}$ is dual to the cross product $\vec{a} \times \vec{b}$. More generally, multiplying a vector by $I$ yields a bivector; multiplying a bivector with $I$ yields a vector.

A general element $\mathrm{M}$ of the algebra $\mathcal{C}_{3}$ is then a sum of the four types. Thanks to the general relation (31) we can write this sum as

$$
\mathrm{M}=a+b I+\vec{a}+I \vec{b},
$$

where the terms represent the scalar, pseudoscalar, vector and bivector parts, respectively, where $a, b$ are scalars, and $\vec{a}, \vec{b}$ are ordinary vectors.

The sum of scalar and pseudoscalar parts is denoted by $\langle\mathrm{M}\rangle_{s}$. Similarly, we denote the sum of vector and bivector parts by $\langle\mathrm{M}\rangle_{v}$. A useful identity is

$$
\langle\mathrm{MN}\rangle_{s}=\langle\mathrm{NM}\rangle_{s},
$$

for any elements $\mathrm{M}$ and $\mathrm{N}$ in $\mathcal{C}_{3}$. Note that the analogous relation for the vector part does not hold. It is also useful to define "real" and "imaginary" parts of elements of $\mathcal{C}_{3}$ by

$$
\operatorname{Re}(\mathrm{M})=\left(\mathrm{M}+\mathrm{M}^{\dagger}\right) / 2 ; \operatorname{Im}(\mathrm{M})=I\left(\mathrm{M}^{\dagger}-\mathrm{M}\right) / 2,
$$

where the $\dagger$ operation, as mentioned above, reverses the order of vectors in a vector product; equivalently, it reverses the sign of $I$ in a decomposition like 32 .

Another involution, of great use in descriptions of relativity, is the Clifford conjugate, which reverses the directions of vectors and pseudovectors but leaves the scalar part the same. We denote the Clifford conjugate by a bar, like so

$$
\overline{\mathrm{M}}=a+b I-\vec{a}-I \vec{b}
$$

for $\mathrm{M}$ given by 32 . 


\section{NON-COVARIANT DESCRIPTION OF ELECTROMAGNETICS WITHIN GA}

\section{A. Preliminaries}

For the description of the EM field we will build on the ideas in the textbook 14, going beyond its treatment in various aspects. This Section gives a non-covariant description of EM, the next presents the covariant version. The results in this Section pertain to general solutions of the free Maxwell equations, not just null fields, except in the very last subsection IV D 1 .

It might be odd to begin this subsection by referring to the standard covariant description of EM, but this will explain why the non-covariant GA description is so elegant; moreover, it demonstrates that the description of EM in terms of the Riemann-Silberstein vector [23] (defined as $\vec{E}+i \vec{B}$ ) is elegant for the simple reason that it is in fact the GA description with $I$ replaced by $i$ (see Eq. (38) below).

The standard covariant description of the EM field is in terms of the antisymmetric tensor $F_{\mu \nu}$ (with the indices running from 0 to 3 ). If we first define the following 4 elements (since they are not all of the same type we use a sans serif symbol to denote them)

$$
\mathrm{e}_{0}=1 ; \mathrm{e}_{1,2,3}=\vec{e}_{1,2,3}
$$

then by defining

$$
\mathrm{F}_{1}=\frac{1}{2} F^{\mu \nu}\left\langle\mathrm{e}_{\mu} \overline{\mathrm{e}}_{\nu}\right\rangle_{v}
$$

(using the usual Einstein convention of implied summation over repeated indices, and using the Clifford conjugate (35)) we get the central result [14]

$$
\mathrm{F}_{1}=\vec{E}+I \vec{B}
$$

Although the object on the left-hand side is covariant, the split into electric and magnetic fields is observerdependent. In fact, $I$ is observer-dependent, because the three spatial unit vectors are.

\section{B. Source-free fields}

We now limit ourselves to a discussion of source-free fields. For such fields we can introduce transverse (and thereby gauge-invariant) vector potentials by

$$
\mathrm{F}_{0}=\vec{A}+I \vec{C}
$$

such that

$$
\mathrm{F}_{1}=\vec{\nabla} \mathrm{F}_{0}
$$

Indeed, when considering separately the four geometric types contained in this equation, we find that the scalar and pseudoscalar parts give $\vec{\nabla} \cdot \vec{A}=0$ and $\vec{\nabla} \cdot \vec{C}=0$, respectively. The sign convention of 39 is such that $\vec{E}=-\vec{\nabla} \times \vec{C}$. The reason for introducing $\vec{C}$ is so we can define an electric helicity. In analogy to 16 we define the electric helicity density

$$
h_{e}=\vec{C} \cdot \vec{E},
$$

and the electric helicity $H_{e}$ is then obtained by integrating this density over all space.

We can inductively define a whole hierarchy of fields and/or potentials by

$$
\mathrm{F}_{n+1}=\vec{\nabla} \mathrm{F}_{n}
$$

for all integer $n$. For example,

$$
\mathrm{F}_{2}=-\vec{\nabla} \times \vec{B}+I \vec{\nabla} \times \vec{E}
$$

For free fields they all satisfy

$$
\left(\partial_{t}+\vec{\nabla}\right) \mathrm{F}_{n}=0
$$

All these equations are then invariant under the duality transformation (4), which is expressed simply as

$$
\mathrm{F}_{0} \mapsto \exp (-I \theta) \mathrm{F}_{0}
$$

This transformation induces the transformation

$$
\mathrm{F}_{n} \mapsto \exp (-I \theta) \mathrm{F}_{n}
$$

Having defined the dagger operation before as reversing the sign of $I$, we also have

$$
\mathrm{F}_{n}^{\dagger} \mapsto \exp (I \theta) \mathrm{F}_{n}^{\dagger}
$$

This makes it straightforward to construct quantities that are invariant under the duality transformation, namely as bilinear quantities containing one term $F_{n}$ and one term $\mathrm{F}_{m}^{\dagger}$ and possibly more factors that are field independent. Such quantities are discussed in the next subsection.

\section{Bilinear quantities}

Here we give the relations between certain bilinear geometric algebraic quantities and the more familiar bilinear vectorial quantities. Since the elements of $\mathcal{C}_{3}$ do not commute in general, it is convenient to consider symmetric and anti-symmetric bilinear quantities. For example, we have

$$
\begin{aligned}
\mathrm{F}_{1}^{\dagger} \mathrm{F}_{1}+\mathrm{F}_{1} \mathrm{~F}_{1}^{\dagger} & =2\left(\vec{E}^{2}+\vec{B}^{2}\right), \\
\mathrm{F}_{1}^{\dagger} \mathrm{F}_{1}-\mathrm{F}_{1} \mathrm{~F}_{1}^{\dagger} & =-4 \vec{E} \times \vec{B}, \\
\operatorname{Re}\left(\mathrm{F}_{1}^{2}\right) & =\vec{E}^{2}-\vec{B}^{2}, \\
\operatorname{Im}\left(\mathrm{F}_{1}^{2}\right) & =2 I \vec{E} \cdot \vec{B} .
\end{aligned}
$$

We can recognize the energy density of the field, the momentum density, and the Lagrangian density in the first three lines. The fourth line gives the quantity that should vanish if a covariant definition of electric and magnetic field 
lines is to be possible, according to 2. Furthermore, we have

$$
\begin{aligned}
\mathrm{F}_{1} \mathrm{~F}_{0}+\mathrm{F}_{0} \mathrm{~F}_{1}= & 2(\vec{E} \cdot \vec{A}-\vec{B} \cdot \vec{C})+ \\
& 2 I(\vec{B} \cdot \vec{A}+\vec{E} \cdot \vec{C}) \\
\mathrm{F}_{1} \mathrm{~F}_{0}-\mathrm{F}_{0} \mathrm{~F}_{1}= & 2 I(\vec{E} \times \vec{A}-\vec{B} \times \vec{C})+ \\
& -2(\vec{B} \times \vec{A}+\vec{E} \times \vec{C})
\end{aligned}
$$

These two definitions are not invariant under duality transformations. Such non-invariant quantities are useful to distinguish quantities that are conserved because of some symmetry from those that are conserved only for particular solutions (see below). Moreover, since measurements on the free EM field may make use of electric charges, measurement can break the duality symmetry, and thus it is not true that only duality-invariant quantities are physically relevant for free fields, as was argued in [24, 25]. A duality-invariant version of Eq. 49 is

$$
\begin{aligned}
\mathrm{F}_{1} \mathrm{~F}_{0}^{\dagger}+\mathrm{F}_{0}^{\dagger} \mathrm{F}_{1}= & 2(\vec{E} \cdot \vec{A}+\vec{B} \cdot \vec{C})+ \\
& 2 I(\vec{B} \cdot \vec{A}-\vec{E} \cdot \vec{C})
\end{aligned}
$$

In the second line the total (i.e., magnetic plus electric) helicity density appears as a pseudoscalar. Combining similar relations yields the magnetic and electric helicity densities separately:

$$
\begin{aligned}
\vec{B} \cdot \vec{A} & =\left(\operatorname{Im}\left(\mathrm{F}_{1}\right) \operatorname{Re}\left(\mathrm{F}_{0}\right)+\operatorname{Re}\left(\mathrm{F}_{0}\right) \operatorname{Im}\left(\mathrm{F}_{1}\right)\right) / 2, \\
\vec{E} \cdot \vec{C} & =\left(\operatorname{Re}\left(\mathrm{F}_{1}\right) \operatorname{Im}\left(\mathrm{F}_{0}\right)+\operatorname{Im}\left(\mathrm{F}_{0}\right) \operatorname{Re}\left(\mathrm{F}_{1}\right)\right) / 2 .
\end{aligned}
$$

We conclude this subsection by noting that duality invariance and its relation to helicity and the separation of the total angular momentum of light into spin and orbital parts has become the subject of very recent studies [? ? ].

\section{Conservation laws for (pseudo)scalar quantities}

We consider here in some detail how one may show within the GA formalism that a (pseudo)scalar quantity is conserved. Take helicity as an example. We use three handy maneuvers. The first is to explicitly take the scalar plus pseudoscalar part of

$$
\begin{aligned}
\partial_{t}\left\langle\mathrm{~F}_{1} \mathrm{~F}_{0}^{\dagger}\right\rangle_{s} & =\left\langle\partial_{t}\left(\mathrm{~F}_{1} \mathrm{~F}_{0}^{\dagger}\right)\right\rangle_{s} \\
& =-\left\langle\vec{\nabla} \mathrm{F}_{1} \mathrm{~F}_{0}^{\dagger}\right\rangle_{s}-\left\langle\mathrm{F}_{1}\left(\vec{\nabla} \mathrm{F}_{0}\right)^{\dagger}\right\rangle_{s} \\
& =-\left\langle\vec{\nabla} \mathrm{F}_{1} \mathrm{~F}_{0}^{\dagger}\right\rangle_{s}-\left\langle\mathrm{F}_{1} \mathrm{~F}_{1}^{\dagger}\right\rangle_{s} .
\end{aligned}
$$

The second maneuver is to note that

$$
\left\langle\vec{\nabla}\left(\mathrm{F}_{1} \mathrm{~F}_{0}^{\dagger}\right)\right\rangle_{s}=\left\langle\vec{\nabla}\left\langle\mathrm{F}_{1} \mathrm{~F}_{0}^{\dagger}\right\rangle_{v}\right\rangle_{s}
$$

because the gradient vector multiplying a scalar term can never give rise to a scalar term. The gradient on the lhs acts on both $F_{1}$ and $F_{0}^{\dagger}$. But because general elements of
$\mathcal{C}_{3}$ do not commute, in order to calculate the result from the gradient acting on the second term, we first move it to the front before taking the derivative. That is, the third maneuver is to write

$$
\mathrm{F}_{1} \mathrm{~F}_{0}^{\dagger}=-\mathrm{F}_{0}^{\dagger} \mathrm{F}_{1}+2\left\langle\mathrm{~F}_{1} \mathrm{~F}_{0}^{\dagger}\right\rangle_{s}
$$

and use this to rewrite

$$
\begin{aligned}
\left\langle\vec{\nabla}\left(\mathrm{F}_{1} \mathrm{~F}_{0}^{\dagger}\right)\right\rangle_{s} & =\left\langle\vec{\nabla} \mathrm{F}_{1} \mathrm{~F}_{0}^{\dagger}-\vec{\nabla}\left(\mathrm{F}_{0}^{\dagger}\right) \mathrm{F}_{1}\right\rangle_{s} \\
& =\left\langle\vec{\nabla} \mathrm{F}_{1} \mathrm{~F}_{0}^{\dagger}+\mathrm{F}_{1}^{\dagger} \mathrm{F}_{1}\right\rangle_{s},
\end{aligned}
$$

where in the last line we used

$$
\vec{\nabla}\left(\mathrm{F}_{n}^{\dagger}\right)=-\left(\vec{\nabla} \mathrm{F}_{n}\right)^{\dagger}
$$

All this together yields

$$
\begin{aligned}
\partial_{t}\left\langle\mathrm{~F}_{1} \mathrm{~F}_{0}^{\dagger}\right\rangle_{s}= & -\vec{\nabla} \cdot\left\langle\mathrm{F}_{1} \mathrm{~F}_{0}^{\dagger}\right\rangle_{v}+\left\langle\mathrm{F}_{1}^{\dagger} \mathrm{F}_{1}\right\rangle_{s} \\
& -\left\langle\mathrm{F}_{1} \mathrm{~F}_{1}^{\dagger}\right\rangle_{s} \\
= & -\vec{\nabla} \cdot\left\langle\mathrm{F}_{1} \mathrm{~F}_{0}^{\dagger}\right\rangle_{v} .
\end{aligned}
$$

This shows that the helicity is conserved for free fields, but so is the quantity

$$
Q=\vec{E} \cdot \vec{A}+\vec{B} \cdot \vec{C}
$$

This quantity $Q$ is not that interesting, however, as it is just proportional to the time derivative of $\vec{A}^{2}+\vec{C}^{2}$ (which we see when substituting the well known relation $\vec{E}=$ $-\partial_{t} \vec{A}$, as well as the less known but similar relation $\vec{B}=$ $\left.-\partial_{t} \vec{C}\right)$, which is itself a conserved quantity.

\section{Helicities and null conditions}

We now wish to consider the magnetic and electric helicities separately. For this purpose it suffices now (given the above results) to consider

$$
\begin{aligned}
\partial_{t}\left\langle\mathrm{~F}_{1} \mathrm{~F}_{0}\right\rangle_{s} & =\frac{1}{2}\left\langle\partial_{t}\left(\mathrm{~F}_{1} \mathrm{~F}_{0}+\mathrm{F}_{0} \mathrm{~F}_{1}\right)\right\rangle_{s} \\
& =-2 \mathrm{~F}_{1}^{2}-\vec{\nabla} \cdot\left\langle\mathrm{F}_{1} \mathrm{~F}_{0}\right\rangle_{v}
\end{aligned}
$$

where above-mentioned maneuvers were used once again. This shows that the magnetic and electric helicities are not individually conserved. But for solutions for which $\mathrm{F}_{1}^{2}=0$, they are individually conserved. Solutions of Maxwell's equations with $\mathrm{F}_{1}^{2}=0$ are termed "null solutions" for obvious reasons, and they have been considered in various contexts both long ago and very recently [17, 26-28] (see also Section VII). Now taking the time derivative of $F_{1}^{2}$ (which is identically zero for a null field, of course) we find

$$
\partial_{t} \mathrm{~F}_{1}^{2}=-2\left\langle\mathrm{~F}_{2} \mathrm{~F}_{1}\right\rangle_{s}
$$


The quantity on the rhs is the difference between two higher-order helicities, not related to the field lines of $\vec{E}$ and $\vec{B}$, but to those of $\vec{\nabla} \times \vec{E}$ and $\vec{\nabla} \times \vec{B}$. Clearly, it vanishes for null fields. (The sum of those higher-order helicities is, in fact, the optical chirality density, which has become of great interest recently [24, 25, 29, 33.)

We can now repeat the story: since the right-hand side must be zero, its time derivative vanishes as well. But that time derivative is now easily found by reference to 60 ,

$$
\begin{aligned}
\partial_{t}\left\langle\mathrm{~F}_{2} \mathrm{~F}_{1}\right\rangle_{s} & =\frac{1}{2}\left\langle\partial_{t}\left(\mathrm{~F}_{2} \mathrm{~F}_{1}+\mathrm{F}_{1} \mathrm{~F}_{2}\right)\right\rangle_{s} \\
& =-2 \mathrm{~F}_{2}^{2}-\vec{\nabla} \cdot\left\langle\mathrm{F}_{2} \mathrm{~F}_{1}\right\rangle_{v} .
\end{aligned}
$$

This way we encounter higher-order versions of the helicity differences, $\left\langle\mathrm{F}_{n+1} \mathrm{~F}_{n}\right\rangle_{s}$, as well as higher-order versions of the null conditions, $\mathrm{F}_{n}^{2}=0$, and they are related through conservation laws.

\section{COVARIANT DESCRIPTION OF ELECTROMAGNETICS WITHIN GA}

\section{A. The Baylis method}

Special relativity can be compactly described in a covariant manner by using the spacetime algebra [13, i.e., the GA constructed from spacetime vectors living in $4 \mathrm{D}$ Minkowski spacetime. However, perhaps surprisingly, $\mathcal{C}_{3}$ can be used just as well for the same purpose. This has been demonstrated by Baylis and coauthors in several articles $[20-22]$. There is a subtle difference between the two descriptions, related to the fact that the spacetime algebra has twice as many basis elements as $\mathcal{C}_{3}$ (it is 16dimensional whereas $\mathcal{C}_{3}$ is 8-dimensional). Physical quantities such as the (rest) mass of a particle or the proper time of a particle in its own rest frame play two roles: as the zeroth component of the energy-momentum four-vector or the spacetime position four-vector, respectively, but also as the Lorentz-invariant length of the respective four-vectors (in any frame, of course). Whereas $\mathcal{C}_{3}$ uses a single representation for either of these two roles, spacetime algebra has two different representations, one for each role. Here we follow Baylis' lead (see also his textbook [14]) and use the simpler algebra $\mathcal{C}_{3}$.

Any four-vector is represented by a "paravector", i.e., a real element of $\mathcal{C}_{3}$. That is, we can write a general paravector as

$$
\mathrm{p}=p_{0}+\vec{p},
$$

with $p_{0}$ a scalar and $\vec{p}$ a $3 \mathrm{D}$ vector. For example, the spacetime four-vector (as defined relative to some arbitrarily chosen fixed origin of Minkowski space) is represented by the paravector

$$
\mathrm{r}=t+\vec{r}
$$

The Minkowski metric is obtained in natural way by defining the "square length" of a paravector to be

$$
\mathrm{p} \overline{\mathrm{p}}=p_{0}^{2}-\vec{p}^{2},
$$

in terms of the Clifford conjugate (35). The minus sign here implies that another important example of a paravector, the gradient four-vector, takes the form

$$
\partial=\partial_{t}-\vec{\nabla} .
$$

The scalar product between different paravectors $\mathrm{p}$ and $\mathrm{q}$ is defined as the scalar part of the product $p \bar{q}$, i.e.

$$
\langle\mathrm{p}, \mathrm{q}\rangle=\langle\mathrm{p} \overline{\mathrm{q}}\rangle_{s}=\frac{1}{2}(\mathrm{p} \overline{\mathrm{q}}+\mathrm{q} \overline{\mathrm{p}}) .
$$

A Lorentz transformation preserves, by definition, this scalar product of paravectors. It acts on paravectors as

$$
\mathrm{p} \mapsto \mathrm{p}^{\prime}=\mathrm{LpL}^{\dagger},
$$

where $L$ can be chosen to satisfy

$$
\mathrm{L} \overline{\mathrm{L}}=1 .
$$

For example, a pure boost is represented as

$$
\mathbf{L}=\exp (\vec{w} / 2),
$$

where $\vec{w}$ is the so-called "rapidity." Compare this to the description of rotations in space, 26) and (27), which are, of course, also elements in the group of Lorentz transformations. Using (69) we also obtain the combined action of Lorentz transformations and involutions:

$$
\begin{aligned}
\overline{\mathrm{p}} & \mapsto \overline{\mathrm{L}}^{\dagger} \overline{\mathrm{p}} \overline{\mathrm{L}} \\
\mathrm{p}^{\dagger} & \mapsto \mathrm{Lp}^{\dagger} \mathrm{L}^{\dagger} .
\end{aligned}
$$

Given the condition (69) on Lorentz transformations, one easily sees that alternating products of paravectors and Clifford conjugates of paravectors transform in a simple way. When there is an odd number of paravectors and conjugates, then the object transforms just as a paravector or as the Clifford conjugate of a paravector. When there is an even number of paravectors and conjugate terms in the alternating product, then it transforms slightly differently. For example, an object $\mathrm{O}=\mathrm{p} \overline{\mathrm{q}}$ transforms as

$$
\mathrm{O} \mapsto \mathrm{O}^{\prime}=\mathrm{LO} \overline{\mathrm{L}}
$$

We can distinguish two contributions to $\mathrm{O}=\mathrm{p} \overline{\mathrm{q}}$ :

$$
\mathrm{O}=\langle\mathrm{p} \overline{\mathrm{q}}\rangle_{s}+\langle\mathrm{p} \overline{\mathrm{q}}\rangle_{v},
$$

where the first term is just the Lorentz-invariant scalar product of $\mathrm{p}$ and $\mathrm{q}$, and for the second type of term (i.e., a sum of vector and pseudovector parts) Baylis uses the name "biparavector."

\section{B. The EM field}

The EM field is described by a biparavector, namely $F_{1}$. The covariant form of the free Maxwell equations is really the same as before:

$$
\bar{\partial} \mathrm{F}_{1}=0 .
$$


We can introduce a gauge potential by

$$
\mathrm{F}_{1}=\langle\partial \overline{\mathrm{A}}\rangle_{v} .
$$

Now here is an interesting difference between the covariant and the non-covariant ways of introducing gauge potentials. Namely, the equivalent of the electric vector potential $\vec{C}$ is defined through

$$
I \mathrm{~F}_{1}=\langle\partial \overline{\mathrm{C}}\rangle_{v}
$$

where $C$ is real, instead of through

$$
\mathrm{F}_{1}=\langle\partial \overline{\mathrm{A}}\rangle_{v}+I\langle\partial \overline{\mathrm{C}}\rangle_{v} \quad \text { (wrong!) }
$$

which one might have thought would be the covariant form of 40 and 39 ).

Since one can pick only 4 linearly independent paravectors, $F_{1}$, like any other biparavector, can be either written as a single product $p \bar{q}$ - and then it is called a simple biparavector-or as a sum of two such products $p \bar{q}+r \bar{s}$. In either case, that decomposition is covariant (unlike the split in electric and magnetic fields). Given a biparavector $F_{1}$ it is easy to check whether it is simple or not: it is simple if and only if $\operatorname{Im}\left(\mathrm{F}_{1}^{2}\right)=0$. In particular, if a field is describable by the covariant generalization of Euler potentials (for a discussion of covariant Euler potentials for a different class of fields, and from a very different perspective, see Ref. 34])

$$
\mathrm{F}_{1}=\langle\partial \alpha \bar{\partial} \beta\rangle_{v},
$$

it must be simple. Given 76 we can introduce covariant electric Euler potentials by

$$
I \mathrm{~F}_{1}=\langle\partial \lambda \bar{\partial} \mu\rangle_{v} .
$$

These two definitions 78 and $(79)$ allow us to define field lines in a covariant way, as follows. A $2 \mathrm{D}$ surface in spacetime defined by setting $\alpha$ and $\beta$ equal to constants (note we use the paravector $r=t+\vec{r}$ as argument here)

$$
\alpha(r)=\alpha_{0} ; \beta(r)=\beta_{0}
$$

is an invariant surface, if we stipulate that $\alpha$ and $\beta$ are scalar fields, i.e., that they transform under Lorentz transformations as

$$
\alpha^{\prime}\left(\mathrm{r}^{\prime}\right)=\alpha(\mathrm{r}) ; \beta^{\prime}\left(\mathrm{r}^{\prime}\right)=\beta(\mathrm{r}) .
$$

(And this we substitute on the left-hand sides of (80).) This invariant surface is swept out over time by a given magnetic field line. Conversely, a magnetic field line in a given inertial frame of reference is then determined by the intersection of the invariant $2 \mathrm{D}$ surface with a spacelike hyperplane, i.e., a hyperplane whose normal is timelike: in other words, it is simply determined by fixing the time coordinate in the given frame in Eq. (80). This then gives the covariant definition of magnetic field lines, and it agrees with that given by Newcomb in [2]. For finding electric field lines in a given frame of reference, we go through the same procedure starting with $(79)$, and end up setting

$$
\lambda(r)=\lambda_{0} ; \mu(r)=\mu_{0}
$$

and fixing the time coordinate in that frame. This procedure then determines what field lines in one reference frame look like in another. Namely, we simply identify magnetic and electric field lines by the pair of constants $\left(\alpha_{0}, \beta_{0}\right)$ and $\left(\lambda_{0}, \mu_{0}\right)$, respectively.

Returning to the definition of helicity density (51) from the previous Section we see it is not covariant, as it is not an alternating product of paravectors and their Clifford conjugates. But it can be turned into a sum of two different covariant terms by inserting an arbitrary field-independent paravector q and its Clifford conjugate (35), like so:

$$
h(\mathrm{q})=\mathrm{F}_{1} \mathrm{q} \mathrm{F}_{0}^{\dagger}+\mathrm{F}_{0}^{\dagger} \overline{\mathrm{q}} \mathrm{F}_{1}=2\left\langle\mathrm{~F}_{1} \mathrm{q} \mathrm{F}_{0}^{\dagger}\right\rangle_{s} .
$$

The first term transforms as a paravector, the second as the Clifford conjugate of a paravector, and their sum is a scalar. We recognize now the helicity density (51) as simply proportional to this scalar. For example, by choosing $\mathbf{q}=\mathrm{u}:=(1,0,0,0)$ it follows that the helicity density equals $h(\mathrm{u}) / 2$. Given this, it is easy to see that the helicity being an integral over all space of the zeroth component of four-vectors is invariant under Lorentz transformations (just like electric charge is, for instance). In fact, this invariance also follows from the expression Eq. (23), which shows the (magnetic) helicity is expressable in terms of the scalar Euler potentials.

\section{NUMERICAL RESULTS}

\section{A. The Hopf-Rañada solutions}

We now apply the results of the preceding Sections to the Hopf-Rañada solutions. Those solutions are usually given in terms of complex functions $\eta$ and $\zeta$ through

$$
\begin{aligned}
\vec{B} & =\frac{1}{4 \pi i} \frac{1}{\left(1+|\eta|^{2}\right)^{2}} \vec{\nabla} \eta \times \vec{\nabla} \eta^{*}, \\
\vec{E} & =\frac{1}{4 \pi i} \frac{1}{\left(1+|\zeta|^{2}\right)^{2}} \vec{\nabla} \zeta \times \vec{\nabla} \zeta^{*} .
\end{aligned}
$$

For completeness, we give them here explicitly

$$
\begin{aligned}
\eta & =\frac{A z+t(A-1)+i(t x-A y)}{A x+t y+i(A(A-1)-t z)} \\
\zeta & =\frac{A x+t y+i(A z+t(A-1))}{t x-A y+i(A(A-1)-t z)}
\end{aligned}
$$

with $A=\left(|\vec{r}|^{2}-t^{2}+1\right) / 2$. But by switching variables from $\eta, \eta^{*}$ to $\phi=\arctan (\operatorname{Re}(\eta) / \operatorname{Im}(\eta))$ and $|\eta|^{2}$ (and similarly for $\zeta$ and $\left.\zeta^{*}\right)$ we get the (non-covariant) Euler form for the magnetic field with

$$
\alpha=\frac{\phi}{2 \pi} ; \beta=\frac{1}{1+|\eta|^{2}},
$$

and a similar result for the electric field in terms of $\zeta$. Remarkably, the same functions also can be used as the covariant Euler potentials. 
The Hopf-Rañada solutions are known to be null fields, i.e., they satisfy both $\vec{E} \cdot \vec{B}=0$ and $|\vec{E}|^{2}=|\vec{B}|^{2}$. We can, therefore, define magnetic and electric field lines covariantly, and both types of field lines move at the speed of light.

The magnetic helicity of these solutions is obtained through Eq. (23) by noting that

$$
\beta_{\max }-\beta_{\min }=1 ; \beta(L)=1 ; \alpha_{\max }-\alpha_{\min }=1,
$$

so that $H_{m}=1$. The same result is obtained in the same way for the electric helicity, $H_{e}=1$. These results had been obtained before in a purely topological way as the index of the Hopf map in [7].

We consider here two $2 \mathrm{D}$ surfaces in $3 \mathrm{D}$ space by considering the locus of points where $\alpha\left(\vec{r}, t_{0}\right)=\alpha_{0}$ at some fixed time $t_{0}$, and, similarly, $\beta\left(\vec{r}, t_{0}\right)=\beta_{0}$. These two surfaces are not invariant. One topological property of these surfaces can be immediately obtained from the character of field lines: the field lines constitute a tangential vector field that is nowhere zero. So it has an index 0 , and therefore, by the Poincaré-Hopf theorem, can only exist on a closed surface if it has Euler characteristic equal to zero. That is, the surface, if it is closed, must be topologically equivalent to a torus. In Fig. 1 we plot a particular surface

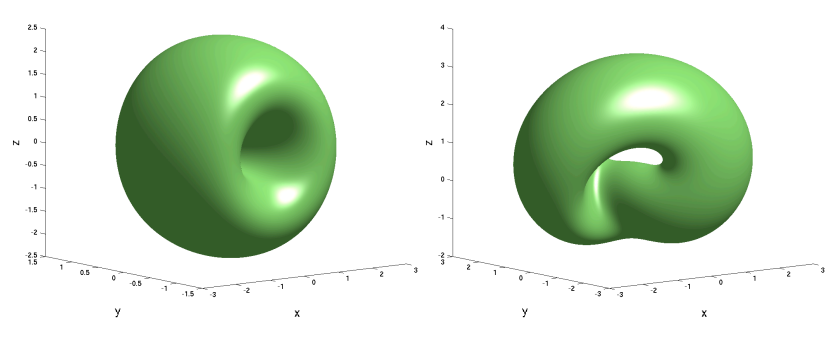

FIG. 1: Surface $\beta_{0}=0.5$ at two different times, $t=0$ on the left and $t=1$ on the right, in the lab frame. Note the axes in left and right plots are not quite the same, and the surface has in fact moved up, i.e., in the positive $z$ direction.

$\beta=$ constant at two different times in the "lab frame," by which we simple mean the frame in which the expressions 85 hold. We pick here and in all similar figures below the value $\beta_{0}=1 / 2$, corresponding to $|\eta|^{2}=1$. The topology of the torus is clearly visible.

Then we plot the same surfaces in two other reference frames, both moving at a speed of $c / 2$, in Figs. 2 and 3 . That is, in those references frames we pick $t^{\prime}=0$ and $t^{\prime}=1$ (while making the standard assumption that the origins in the different frames coincide at $t=t^{\prime}=0$ ), and we keep the constant $\beta_{0}=1 / 2$ the same. The plots confirm that the Lorentz-transformed surfaces stay closed and topologically

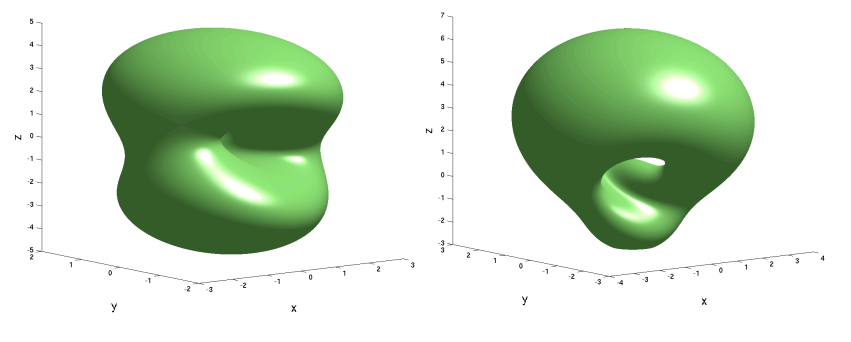

FIG. 2: Same as Fig. 1 but in a reference frame moving in the positive $z$ direction with speed $c / 2$.

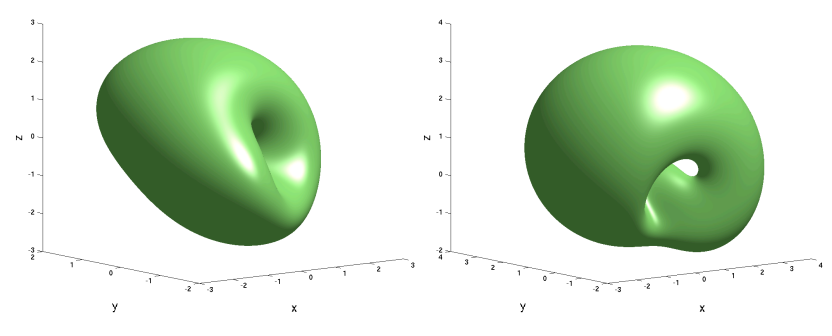

FIG. 3: Same as Fig. 1 but in a frame moving in the positive $x$ direction with speed $c / 2$.

equivalent to a torus. We note that the surfaces are not stationary, so the deformations cannot be explained simply from just length contraction.

In Figs. 46 we then plot surfaces $\alpha=\alpha_{0}$ constant, again in the same three reference frames as before, and at the same times. The constant is picked the same in the three figures, namely $\alpha_{0}=0.1 /(2 \pi)$, so that we can say we plot the same surfaces as seen by different observers. These surfaces are not closed, but the figures do show they have one handle.

In the last set of three figures, 7 , 8 and 9 we plot the intersections of the surfaces shown in the previous six figures. That is, we plot the magnetic field lines corresponding to the two constants $\beta_{0}=0.5$ and $2 \pi \alpha_{0}=0.1$. In addition we plot in the same figures the electric field line corresponding to the intersection of surfaces $\lambda=\lambda_{0}$ and $\mu=\mu_{0}$ with the constants chosen the same as for the magnetic case, i.e., 

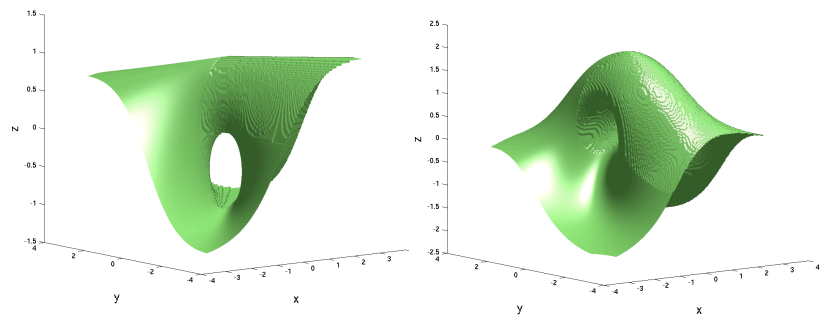

FIG. 4: Surface $2 \pi \alpha_{0}=0.1$ at two different times, $t=0$ on the left and $t=1$ on the right, in the lab frame. Note the axes in left and right plots are not quite the same, and the surface has in fact moved up, i.e., in the positive $z$ direction.

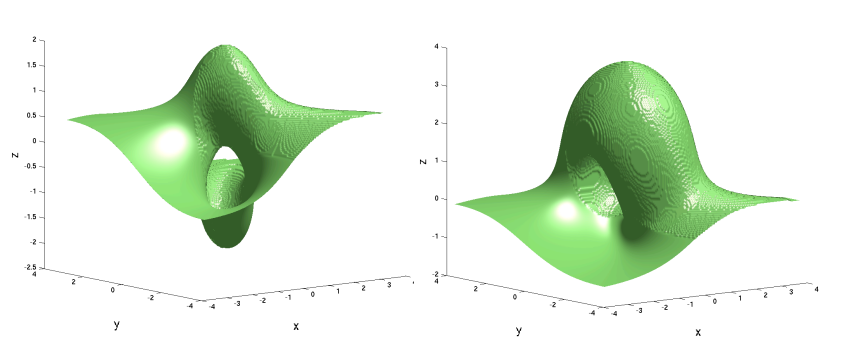

FIG. 5: Same as Fig. 4 but in a reference frame moving in the positive $z$ direction with speed $c / 2$.

$\lambda_{0}=\alpha_{0}$ and $\mu_{0}=\beta_{0}$.

\section{B. Extension of Hopf-Rañada solutions}

Ranãda has given an extension of his solutions. Namely, given the complex function $\eta$, we can pick, according to 8 the following pair of Euler potentials

$$
\begin{aligned}
\alpha_{n} & =\frac{1}{2 \pi} \arctan \left(\frac{\operatorname{Re}\left(\eta^{n}\right)}{\operatorname{Im}\left(\eta^{n}\right)}\right) \\
\beta & =\frac{1}{1+|\eta|^{2}}
\end{aligned}
$$

for any integer $n$. That is, the function $\eta \equiv|\eta| \exp (i \phi)$ in (84) is replaced by the function $\eta_{n}=|\eta| \exp (n i \phi)$.

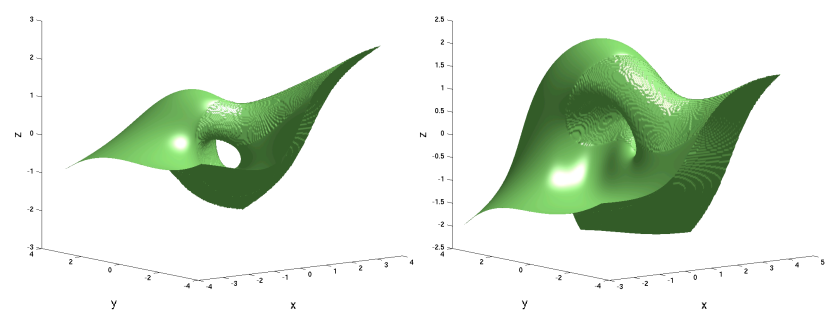

FIG. 6: Same as Fig. 4 but in a frame moving in the positive $x$ direction with speed $c / 2$.

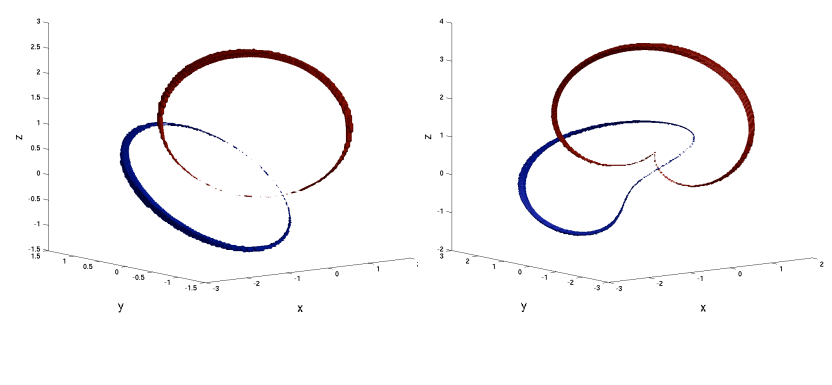

FIG. 7: In blue: magnetic field lines in the lab frame obtained as intersections of the surfaces plotted in Figs. 1 and 4 The other field line (red) is electric, and is obtained with the same procedure applied to the surfaces $\lambda=\lambda_{0}=0.1 /(2 \pi)$ and $\mu=$ $\mu_{0}=0.5$. On the left $t=0$, on the right $t=1$.

But these solutions are really the same: after all, setting $\eta_{n}$ equal to a (complex) constant is equivalent to setting $\eta$ equal to a (different, complex) constant, and so the field lines of $\eta$ and $\eta_{n}$ are the same. Rañada does state that the magnetic helicity changes by a factor of $n^{2}$, but that arises trivially from the simple relations $\vec{B}_{n}=n \vec{B}_{1}$ and $\vec{A}_{n}=n \vec{A}_{1}$.

Whereas surfaces $\alpha=$ constant are manifolds (even if not simply connected), surfaces $\alpha_{n}=$ constant for $n>1$ are not, and consist of $n$ branches. We plot an example in Fig. 10, where we chose $n=3$ at time $t=0$, and the constant equals $0.3 /(2 \pi)$. Three branches are clearly visible. These three branches correspond to three different surfaces obtained by setting $\alpha$ equal to three constants $\alpha_{0}=0.1 /(2 \pi)$ and $\alpha_{0} \pm 1 / 3$. Note that these branches may 

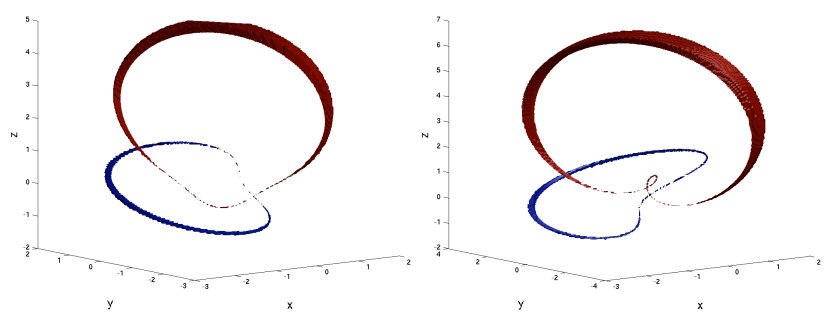

FIG. 8: Same as Fig. 7 but in a reference frame moving in the positive $z$ direction with speed $c / 2$.

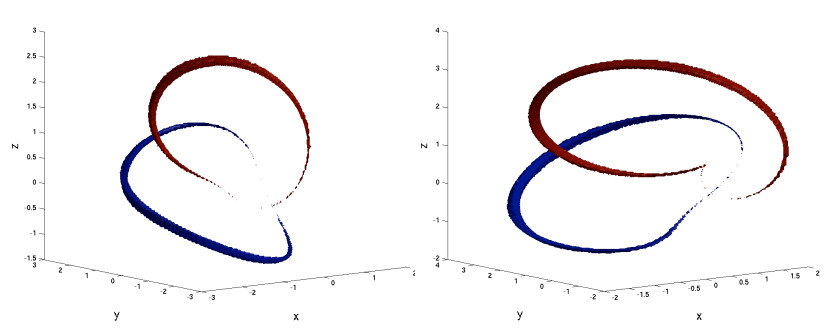

FIG. 9: Same as Fig. 7 but in a frame moving in the positive $x$ direction with speed $c / 2$.

intersect at most at the points where $|\eta|^{2}=0$, the branch cut of the function $\alpha_{n}$ (here, the $x$ axis). One can see in the plot that at those points the gradient of $\alpha_{n}$ is singular. The field line obtained by intersecting the branches with the surface $\beta=0.5$ is plotted in Fig. 11. That plot shows that this is not a single field line, but three field lines.

\section{THE BATEMAN SOLUTIONS}

In his book from 1915 36, Bateman constructs a broad class of solutions to the free Maxwell equations that all satisfy the null property, $F_{1}^{2}=0$. Recall that for such solutions electric and magnetic helicities are conserved, and field lines can be defined covariantly. The description of those solutions in the book comes tantalizingly close to the description used in the present paper, with one important

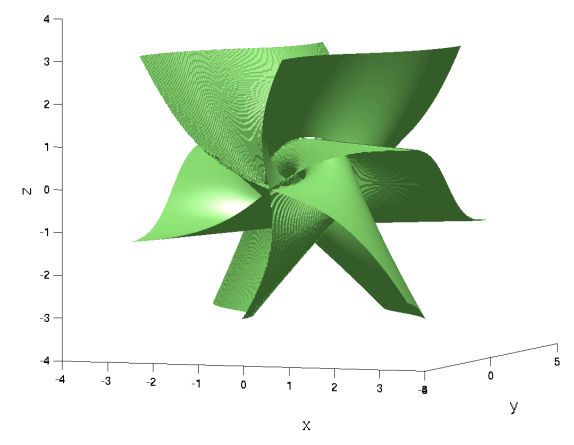

FIG. 10: Plot of surface of constant $\alpha_{3}=0.3 /(2 \pi)$ at time $t=0$ in the lab frame. The multi-valued function $\alpha_{3}$ has 3 branches, which may intersect at most in points where the gradient is singular. The viewing angle is different here than in previous figures, so as to make the structure of the corresponding field line(s) more clearly visible (see next figure).

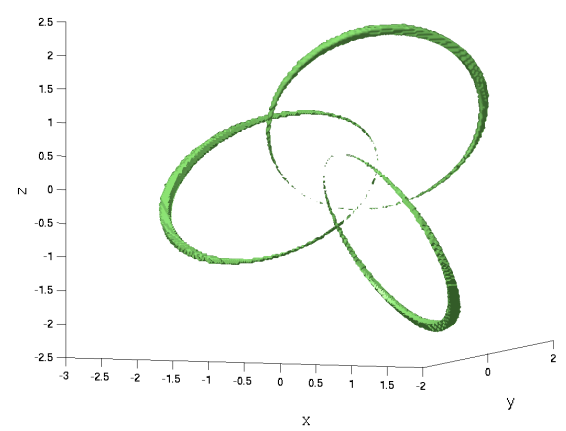

FIG. 11: Magnetic field lines as determined by the intersection of the surface plotted in the preceding figure with the surface $\beta=0.5$.

difference: Bateman uses complex Euler potentials, instead of real ones. This does negate their advantage in finding field lines or expressing the helicity. The complex solutions satisfy an additional constraint, which may help in constructing real Euler potentials, but we have not succeeded in doing so. Even though Bateman's solutions are not given in covariant form in his book, it is straightforward to convert to covariant notation.

In more detail then, Bateman finds pairs of complex functions $\phi_{1}$ and $\phi_{2}$, such that (using the lower of the ambiguous sign he uses in his Eqs. (2) and (10))

$$
\mathrm{F}_{1}=\left\langle\partial \phi_{1} \bar{\partial} \phi_{2}\right\rangle_{v} \text {. }
$$

Moreover, these functions satisfy the condition

$$
\left\langle\bar{\partial} \phi_{1} \partial \phi_{2}\right\rangle_{v}=0 .
$$

This constraint implies $\mathrm{F}_{1}^{2}=0$, as can be easily checked within the formalism of GA.

Examples of pairs of functions $\phi_{1,2}$ can be obtained by choosing $\phi_{1}=\beta_{B}$ and $\phi_{2}=\alpha_{B} / 2$, where $\alpha_{B}$ and $\beta_{B}$ 
(conforming to the notation Bateman uses) must be picked with the lower sign in his Eqs. (11) or (13)

$$
\begin{aligned}
& \phi_{1}=x \sin \vartheta-y \cos \vartheta-t \\
& \phi_{2}=\frac{1}{2}(x \cos \vartheta+y \sin \vartheta+i z),
\end{aligned}
$$

where $\vartheta$ is a parameter that can be chosen arbitrarily, or

$$
\begin{aligned}
\phi_{1} & =r-t \\
\phi_{2} & =\frac{x+i y}{2(z+r)} .
\end{aligned}
$$

The Hopf-Rañada solutions are, in fact, a special example of the Bateman solutions, as has been shown in [26]. Even this correspondence, where both Hopf-Rañada and Bateman solutions are explicitly known, is not easy to demonstrate explicitly.

\section{SUMMARY}

We described a known set of solutions to the free Maxwell equations, the Hopf-Rañada linked and knotted fields, in a compact geometric way by using Geometric Algebra. In particular, we represented the solutions in covariant form as

$$
\mathrm{F}_{1}=\langle\partial \alpha \bar{\partial} \beta\rangle_{v}
$$

with $\alpha$ and $\beta$ real scalar potentials. This way of writing yields magnetic field lines as the intersections of two such $2 \mathrm{D}$ surfaces $\alpha=\alpha_{0}$ and $\beta=\beta_{0}$. We plotted examples of these 2D surfaces and their Lorentz-transformed versions, confirming that such surfaces must have genus equal to 1 . Moreover, this description shows that whereas ordinarily field lines cannot be linked, they can when $\alpha$ is multivalued and its gradient is singular. The electric field lines are obtained by writing, similarly,

$$
I \mathrm{~F}_{1}=\langle\partial \lambda \bar{\partial} \mu\rangle_{v}
$$

with $I$ the unit pseudoscalar volume element (so that the left-hand side corresponds to the dual of $F_{1}$ ). For the HopfRañadasolutions, we showed explicitly that electric and magnetic helicities are individually conserved in time; that helicity is invariant under Lorentz transformations, and that their chirality vanishes, all as a consequence of their "null property" $F_{1}^{2}=0$. Finally, we showed that Bateman's solutions, which have the null property as well, can be written in the form

$$
\mathrm{F}_{1}=\left\langle\partial \phi_{1} \bar{\partial} \phi_{2}\right\rangle_{v},
$$

in terms of complex potentials that satisfy

$$
\left\langle\bar{\partial} \phi_{1} \partial \phi_{2}\right\rangle_{v}=0
$$

The question how these solutions can be rewritten in the form (93) remains open.

\section{Acknowledgements}

I thank Dirk Bouwmeester for his hospitality and discussions on the Hopf-Rañada solutions and Geometric Algebra with his group during a 6-weeks' visit to Leiden University
[1] N. Nersessian, Faraday to Einstein: Constructing meaning in scientific theories (Springer, 1984).

[2] W. Newcomb, Annals of Physics 3, 347 (1958).

[3] D. P. Stern, Am. J. Phys. 38, 494 (1970).

[4] H. Moffatt, J. Fluid Mech 35, 117 (1969).

[5] M. Berger and G. Field, Journal of Fluid Mechanics 147, 133 (1984).

[6] A. Rañada, European Journal of Physics 13, 70 (1992).

[7] A. Rañada, Journal of Physics A: Mathematical and General 25, 1621 (1992).

[8] A. Rañada and J. Trueba, Physics Letters A 232, 25 (1997).

[9] J. Trueba and A. Rañada, European Journal of Physics 17, 141 (1999).

[10] W. Irvine and D. Bouwmeester, Nature Physics 4, 716 (2008).

[11] W. Irvine, Journal of Physics A: Mathematical and Theoretical 43, 385203 (2010).

[12] D. Hestenes, American Journal of Physics 71, 104 (2003).

[13] D. Hestenes, American Journal of Physics 71, 691 (2003).

[14] W. Baylis, Electrodynamics: A Modern Geometric Approach, Progress in Mathematical Physics (Birkhäuser Boston, 1998).

[15] C. Doran and A. Lasenby, Geometric algebra for physicists (Cambridge University Press, 2003).

[16] R. Abłamowicz and G. Sobczyk, Lectures on Clifford (ge- ometric) algebras and applications (Birkhauser, 2004).

[17] H. Bateman, The mathematical analysis of electrical and optical wave-motion on the basis of Maxwell's equations: by H. Bateman... (University press, 1915).

[18] V. S. Semenov, D. B. Korovinski, and H. K. Biernat, Nonlinear Processes in Geophysics 9, 347 (2002).

[19] J. Dungey, Monthly Notices of the Royal Astronomical Society 113, 679 (1953).

[20] W. Baylis and G. Jones, Journal of Physics A: Mathematical and General 22, 1 (1989).

[21] W. Baylis, Clifford (Geometric) Algebras with Applications in Physics, Mathematics and Engineering 1, 237 (1996).

[22] W. Baylis and G. Sobczyk, International Journal of Theoretical Physics 43, 2061 (2004).

[23] I. Bialynicki-Birula and Z. Bialynicka-Birula, Optics Communications 264, 342 (2006).

[24] R. Cameron, S. Barnett, and A. Yao, New Journal of Physics 14, 053050 (2012).

[25] S. Barnett, R. Cameron, and A. Yao, Physical Review A 86, 013845 (2012).

[26] I. Besieris and A. Shaarawi, Optics Letters 34, 3887 (2009).

[27] I. Bialynicki-Birula, Journal of Optics A: Pure and Applied Optics 6, S181 (2004).

[28] I. Besieris and A. Shaarawi, Progress In Electromagnetics Research B 8, 1 (2008). 
[29] Y. Tang and A. Cohen, Physical Review Letters 104, 163901 (2010).

[30] Y. Tang and A. Cohen, Science 332, 333 (2011).

[31] E. Hendry, T. Carpy, J. Johnston, M. Popland, R. Mikhaylovskiy, A. Lapthorn, S. Kelly, L. Barron, N. Gadegaard, and M. Kadodwala, Nature Nanotechnology 5, 783 (2010).

[32] K. Bliokh and F. Nori, Physical Review A 83, 021803 (2011).

[33] M. Coles and D. Andrews, Physical Review A 85, 063810
(2012).

[34] T. Uchida, Phys. Rev. E 56, 2181 (1997).

[35] J. Earman and C. Glymour, Historical Studies in the Physical Sciences 11, 49 (1980).

[36] The references to the Aether theory in this book are, with hindsight, amusing and puzzling, since the book was written 10 years after 1905. Those references become more understandable after reading 35 . 